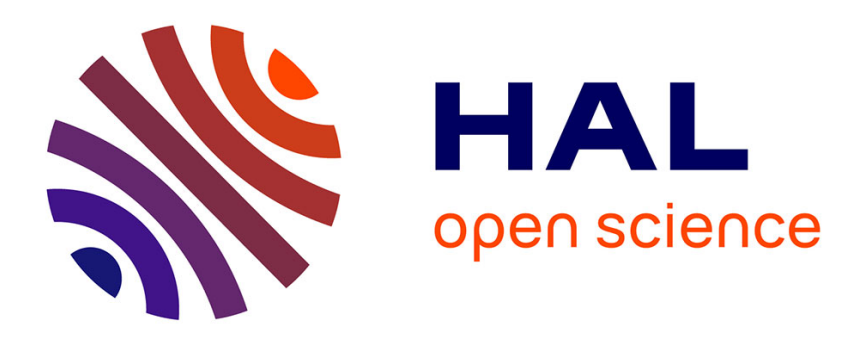

\title{
Injectivity of the inverse optimal control problem for control-affine systems
}

Frédéric Jean, Sofya Maslovskaya

\section{To cite this version:}

Frédéric Jean, Sofya Maslovskaya. Injectivity of the inverse optimal control problem for controlaffine systems. 2019 IEEE Conference on Decision and Control (CDC), Dec 2019, Nice, France. 10.1109/CDC40024.2019.9028877 . hal-02089575

\section{HAL Id: hal-02089575 \\ https://hal-ensta-paris.archives-ouvertes.fr/hal-02089575}

Submitted on 3 Apr 2019

HAL is a multi-disciplinary open access archive for the deposit and dissemination of scientific research documents, whether they are published or not. The documents may come from teaching and research institutions in France or abroad, or from public or private research centers.
L'archive ouverte pluridisciplinaire HAL, est destinée au dépôt et à la diffusion de documents scientifiques de niveau recherche, publiés ou non, émanant des établissements d'enseignement et de recherche français ou étrangers, des laboratoires publics ou privés. 


\title{
Injectivity of the inverse optimal control problem for control-affine systems
}

\author{
Frédéric Jean $^{1}$ and Sofya Maslovskaya ${ }^{2}$
}

\begin{abstract}
Given a control system and a set of optimal trajectories, is it possible to recover the cost for which the trajectories are minimizing? This question is called inverse optimal control problem, and the problem is said to be injective when it admits a unique solution. In this paper we present a general approach to address the issue of the cost uniqueness in the class of quadratic costs and in the case of dynamics given by a control-affine system. We then apply this method to characterize the non-uniqueness cases for a special subclass of control-affine systems.
\end{abstract}

\section{INTRODUCTION}

Inverse optimal control problem has attracted renewed interest in the last 10 years due to the growing number of its application, in particular, in modeling the human movements and in the field of humanoid robotics. Actually, one of the dominant hypothesis in physiology for describing human movements is that the realized movements of the mechanical system minimize some cost function under dynamics constraints (see [28], [27] for instance). However, even if it is known that a movement is optimal, the criteria being optimized is hidden. Thus, to model the human movements we should first solve an inverse optimal control problem: given the data of the realized movements and the dynamic constraints (i.e. the control system), find the cost function with respect to which the movements are optimal. This approach has already proved to be useful in the study of the human locomotion [8], [22] and the arm movements [4], [5]. In robotics, inverse optimal control is a tool to get the most adapted cost function to produce for instance biomimetic motions for humanoid robots [2], [17], or to predict human actions for autonomous robots [14], [15]. However, while in robotics we can be satisfied with numerical and approximate resolution methods, the modeling of human movement requires a more qualitative approach to understand the phenomena involved. It is therefore necessary to carry out a theoretical study of the problem.

From a mathematical point of view, inverse optimal control problems belong to the class of inverse problems where the first issue is whether the problem is well posed. Formally, given a dynamic and a class of candidate cost functions, for the corresponding direct optimal control problems we can define an operator which maps a cost function to the optimal synthesis, i.e. to the set of all optimal trajectories of the corresponding optimal control problem, for all realizable

\footnotetext{
${ }^{1} \mathrm{~F}$. Jean is with the Applied Mathematics department of ENSTA ParisTech, F-91120 Palaiseau, France. frederic.jean@ensta-paristech.fr

${ }^{2}$ S. Maslovskaya is with INRIA, B.P. $93-06902$ Sophia Antipolis cedex, France. sofya.maslovskayaeinria.fr
}

initial and final points. The inverse optimal control problem is to inverse this operator. For such an inverse problem to be well posed, it should be surjective, injective and stable. In applications surjectivity is usually assumed to be satisfied, and the first issue is the injectivity, i.e. is there a one to one correspondence between the costs and the optimal syntheses?

In control theory, inverse optimal control was first introduced by Kalman in 1964 [12] for linear-quadratic regulators. Many authors have addressed this issue then (see [13] for a detailed review), and we finally made a complete study of the injectivity of the inverse optimal control in linearquadratic case in [9]. Note that before the definition of Kalman, the inverse problem was already extensively studied in the domain of the calculus of variation (see [24] for a review), but these studies focus on the surjectivity of the inverse problem, not on the injectivity. After the paper of Kalman, the inverse problem was stated for different classes of optimal control problems: for nonlinear [26], [18], discrete [20], [25], stochastic (Markov decision processes [19], [23]) cases and others. Nevertheless, even in the continuous deterministic case, no general methodology emerges, mainly because the inverse problem with costs of general form is highly ill-posed, as noted in [21].

In view of these results, it is legitimate to reduce the class of candidate cost functions, typically by restricting to the class of integral costs that are quadratic with respect to control. For this class, we developed a new approach based on the concept of orbital diffeomorphism, which allowed us to make a very complete study of the case where the dynamics is a control-affine system without drift [10], [11] (see also [29]). The aim of the present paper is to extend this approach to the case of general control-affine systems.

Our approach (both in [10], [11] and in [9]) is based on one guideline and two technical steps. The guideline is that non injectivity of the inverse problem only appears when the control system admits a particular product structure. The first technical step is to show that the data of the inverse problem, the minimizers, can be transferred at the level of the Pontryagin Hamiltonian equations near appropriate trajectories, called ample geodesics. This idea allows to define the notion of orbital diffeomorphisms, which is our main tool to exhibit the product structure. The second step consists in replacing the control system by a nilpotent approximation when analyzing the equations of the orbital diffeomorphism.

For the study of control-affine systems, we keep the same guideline and we seek to use the same technical steps. Compared to the driftless case, however, we face new difficulties since there are no general results on ample geodesics and 
no good notion of nilpotent approximation. Thus we had to develop the corresponding tools, which is done in the $\mathrm{PhD}$ thesis [16]. Since these developments are long and technical, they are presented in this paper without proof or details, for which we refer to [16] (they will be the subject of a future paper). Then we show how to apply these tools in the first treatable case, the one with $n-1$ controls ( $n$ is the dimension of the state space).

The paper is organized as follows. Sect. II presents the main definitions and introduce the notion of equivalent costs, which allows us to study the injectivity of the inverse problem. We give in Sect. III the results on ample geodesics, orbital diffeomorphisms, and on the link between the latter concept and cost equivalence, and in Sect. IV the construction of the nilpotent approximation and its consequence on the analysis of ample geodesics and orbital diffeomorphism. Finally we study in Sect. V the case with $n-1$ controls.

\section{INVERSE OPTIMAL CONTROL PROBLEMS}

\section{A. Inverse problem and injectivity}

Fix a smooth $n$-dimensional manifold $M$ and a controlaffine system on $M$ given by

$$
\dot{q}=f_{0}(q)+\sum_{i=1}^{m} u_{i} f_{i}(q), \quad u=\left(u_{1}, \ldots, u_{m}\right) \in \mathbb{R}^{m},
$$

where $f_{0}, f_{1}, \ldots, f_{m}$ are smooth vector fields on $M$. We denote by $q_{u}$ a trajectory of this system. The choice of a criterion

$$
J\left(q_{u}\right)=\int_{0}^{T} L\left(q_{u}(t), u(t)\right) d t,
$$

defines the following class of direct optimal control problems: given a pair of points $q_{0}, q_{1}$ and a final time $T>0$, determine

$$
\inf \left\{J\left(q_{u}\right): q_{u} \text { traj. of }(1), q_{u}(0)=q_{0}, q_{u}(T)=q_{1}\right\} .
$$

The corresponding inverse optimal control problem arises as follows: given the dynamics (1) and a set $\Gamma$ of trajectories, find a cost $L$ such that every $\gamma \in \Gamma, \gamma:[0, T] \rightarrow M$, is a solution of the optimal control problem (2) associated with $q_{0}=\gamma(0)$ and $q_{1}=\gamma(T)$.

We consider in this paper the question of the injectivity of the inverse problem: does the set of minimizers of (2) (for all $q_{0}, q_{1} \in M$ and $T>0$ ) determine in a unique way the cost $L$ in a chosen class of costs $\mathcal{L}$ ? The uniqueness is meant "up to a multiplicative constant", since two constantly proportional costs $c L$ and $L$ trivially define the same set of minimizers.

We choose here to consider this problem in the class of quadratic costs (note however that most of our results can easily be extended to the class of Tonelli Lagrangians).

Definition 2.1: The class $\mathcal{L}$ is the set of quadratic costs

$$
L(q, u)=u^{T} R(q) u
$$

where, for any $q \in M, R(q)$ is a symmetric positive definite $(m \times m)$ real valued matrix, and the mapping $q \mapsto R(q)$ is smooth.
We make moreover the following hypothesis on the control system.

Assumption 1: The vector fields $f_{0}, f_{1}, \ldots, f_{m}$ satisfy:

- the weak Hörmander condition at any $q \in M$, i.e.,

$$
\operatorname{Lie}_{q}\left(\left\{\left(\operatorname{ad} f_{0}\right)^{s} f_{i}: s \geq 0, i=1, \ldots, m\right\}\right)=T_{q} M ;
$$

- the points $q$ such that $\operatorname{rank}\left\{f_{0}, f_{1}, \ldots, f_{m}\right\}(q)=m+1$, called regular points, form an open dense subset of $M$.

The first condition ensures that the optimal control problem (2) has a non trivial set of solutions. The second one guarantees that the system can never be reduced locally to a driftless control system.

We will study the injectivity through the following equivalence relation on $\mathcal{L}$.

Definition 2.2: We say that two costs $L$ and $\tilde{L}$ in $\mathcal{L}$ are

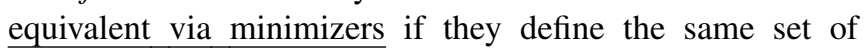
minimizers of (2).

The existence of two non-proportional costs in $\mathcal{L}$ which are equivalent via minimizers implies that the inverse optimal control problem may not have a unique solution, i.e. that the inverse problem is not injective.

\section{B. Example of non-trivially equivalent costs}

Let us construct an example of non proportional costs having the same minimizers. Fix positive integers $N$ and $m_{i} \leq n_{i}$ for $i=1, \ldots, N$, and set $m=\sum_{i} m_{i}, n=\sum_{i} n_{i}$. We denote the coordinates on $\mathbb{R}^{n}=\mathbb{R}^{n_{1}} \times \cdots \times \mathbb{R}^{n_{N}}$ by $x=\left(\bar{x}_{1}, \ldots, \bar{x}_{N}\right)$, where $\bar{x}_{j}=\left(x_{j}^{1}, \ldots, x_{j}^{n_{j}}\right)$, and similarly the coordinates on $\mathbb{R}^{m}$ by $u=\left(\bar{u}_{1}, \ldots, \bar{u}_{N}\right)$. We consider an affine-control system on $\mathbb{R}^{n}$ of the form:

$$
\left\{\begin{array}{c}
\dot{\bar{x}}_{1}=f_{0}^{1}\left(\bar{x}_{1}\right)+\sum_{i=1}^{m_{1}} u_{1}^{i} f_{i}\left(\bar{x}_{1}\right), \\
\vdots \\
\dot{\bar{x}}_{N}=f_{0}^{N}\left(\bar{x}_{N}\right)+\sum_{i=1}^{m_{N}} u_{N}^{i} f_{i}\left(\bar{x}_{N}\right),
\end{array}\right.
$$

and a cost of the form

$$
L(x, u)=\sum_{i=1}^{N} \bar{u}_{i}^{T} R_{i}\left(\bar{x}_{i}\right) \bar{u}_{i}
$$

The corresponding optimal control problem appears as a juxtaposition of $N$ different problems that can be treated separately, and its solution is the product of the $N$ corresponding solutions. Thus, for any $N$-tuple $\alpha_{1}, \ldots, \alpha_{N}$ of positive real numbers, the cost

$$
\tilde{L}(x, u)=\sum_{i=1}^{N} \alpha_{i} \bar{u}_{i}^{T} R_{i}\left(\bar{x}_{i}\right) \bar{u}_{i}
$$

is equivalent via minimizers to $L$, and $\tilde{L}$ is non-proportional to $L$ if the $\alpha_{i}$ 's are not all equal to each other.

This example suggest to introduce the following definition.

Definition 2.3: We say that an optimal control problem defined by (1) and (3) admits a product structure near $q_{0} \in$ $M$ if there exist local coordinates $x$ in which the dynamics and the cost take the form (5) and (6) respectively.

We actually conjecture that, when a cost in $\mathcal{L}$ admits a non proportional equivalent cost, then the corresponding problem has necessarily this product structure. The purpose of this paper is to move towards this conjecture. 


\section{Cost equivalence via geodesics}

Pontryagin Maximum Principle gives a necessary conditions for a curve to be minimizing for the optimal control problem (2). Recall that an extremal of (2) is a curve $(q, p)(\cdot)$ on $T^{*} M$ solution of the Hamiltonian equations arising from the maximization w.r.t. $u$ of

$$
H\left(q, p, u, p^{0}\right)=\left\langle p, f_{0}(q)+\sum_{i=1}^{m} u_{i} f_{i}(q)\right\rangle+p^{0} L(q, u),
$$

where $p^{0} \leq 0$ is a scalar. There are two kinds of extremals, the normal and abnormal ones, corresponding to $p^{0}=-\frac{1}{2}$ and $\overline{p^{0}=0}$ respectively. The projection of a normal (resp. abnormal) extremal onto $M$ is called a normal (resp. abnormal) geodesic. A geodesic can be both normal and abnormal, and it is called strictly normal when it is a projection only of normal extremals.

Definition 2.4: We say that two costs $L$ and $\tilde{L}$ are equivalent via geodesics if the corresponding classes of optimal control problems have the same geodesics.

Remark 2.5: Abnormal geodesics depend only on the control system and are independent of the cost. So, they are the same for all costs in our class and are irrelevant for the inverse problem. As a consequence, for two costs to be equivalent via geodesics we only need the costs to have the same strictly normal geodesics.

Let $h(q, p)=\max _{u} H\left(q, p, u,-\frac{1}{2}\right)$ be the (maximized) normal Hamiltonian. Then the normal geodesics are the projections on $M$ of the integral curves of the Hamiltonian flow, i.e. $\gamma(t)=\pi\left(e^{t \vec{h}} \lambda\right)$ for $\lambda \in T^{*} M$. Moreover, by the strong Legendre condition normal geodesics are minimizers for small time [7, Th. 1.6], thus the same technique as in the sub-Riemannian case in [10] allows to prove the following Lemma.

Lemma 2.6: If two costs in $\mathcal{L}$ are equivalent via minimizers, then they are equivalent via geodesics.

Lemma 2.6 permits to reduce the study of equivalence via minimizers to the study of equivalence via geodesics.

Corollary 2.7: If there do not exist pairs of non proportional costs in $\mathcal{L}$ that are equivalent via geodesics, then the inverse optimal control problem is injective.

\section{ORBITAL DIFFEOMORPHISM}

We have seen that we can replace minimizers by geodesics in the study of equivalence of costs. We show now that the equivalence via geodesics extends to a relation between the normal Hamiltonians called orbital diffeomorphism. As stated in the introduction, the results are given without proof, for which we refer to [16, Chap. $1 \& 4$ ].

\section{A. From geodesics to extremals}

Let $q_{u}(\cdot)$ be a geodesic of (2) defined on $[0, T]$, and consider the linearized system around $q_{u}$ in some local coordinates $x$,

$$
\dot{x}(t)=A(t) x(t)+B(t) v(t)
$$

where the matrices $A(t)$ and $B(t)$ are defined as

$$
\begin{aligned}
& A(t)=\frac{\partial f_{0}}{\partial x}\left(q_{u}(t), u(t)\right)+\sum_{i=1}^{m} u_{i} \frac{\partial f_{i}}{\partial x}\left(q_{u}(t), u(t)\right), \\
& B(t)=\left(\begin{array}{lll}
f_{1}\left(q_{u}(t)\right) & \cdots & \left.f_{m}\left(q_{u}(t)\right)\right)
\end{array}\right) .
\end{aligned}
$$

Consider the sequence of matrix $B_{i}, i \in \mathbb{N}$, defined by

$$
B_{0}(t)=B(t), \quad B_{i+1}(t)=A(t) B_{i}(t)-\frac{d B_{i}(t)}{d t} .
$$

Definition 3.1: A geodesic $q_{u}$ is ample at $t$ if and only if there holds $\operatorname{span}\left\{B_{i}(t) v: v \in \overline{R^{m}}, i \in \mathbb{N}\right\}=\mathbb{R}^{n}$. A covector $\lambda \in T^{*} M$ is ample if the geodesic $q_{u}(t)=\pi\left(e^{t \vec{h}} \lambda\right)$ is ample at $t=0$.

Notice that if a geodesic is ample at $t=0$, then it is strictly normal on any small enough interval $[0, \varepsilon]$ (see [1, Prop. 3.6]). Ample geodesics play a crucial role in the study of equivalence of costs because they are the geodesics characterized by their jets. Let us precise this fact. Fix a nonnegative integer $k$. For a given curve $\gamma: I \rightarrow M, I \subset \mathbb{R}$, denote by $j_{t_{0}}^{k} \gamma$ the $k$-jet of $\gamma$ at the point $t_{0}$. Given $q \in M$, we denote by $J_{q}^{k}(L)$ the space of $k$-jets at $t=0$ of the normal geodesics of $L$ issued from $q$. We set $J^{k}(L)=\bigsqcup_{q \in U} J_{q}^{k}(L)$ and we define the maps $P^{k}: T^{*} M \mapsto J^{k}(L)$, by

$$
P^{k}(\lambda)=j_{0}^{k} \pi\left(e^{t \vec{h}} \lambda\right)
$$

Lemma 3.2: Let $\lambda \in T^{*} M$ be ample. Then there exists an integer $k_{0}$ such that the map $P^{k_{0}}$ is an immersion at $\lambda$.

Thus the existence of ample geodesics is crucial to recover the extremals from the geodesics. In the driftless case, i.e. when $f_{0} \equiv 0$, the existence of ample geodesics was proven in [1]. For proper control-affine systems, the existence of an ample geodesic starting at a given point is still an open question except in the analytic case, where this existence can be deduced from [3, Theorem 2].

Theorem 3.3: Assume that the manifold $M$, the vector fields $f_{0}, \ldots, f_{m}$, and the cost $L$ are analytic, and that Assumption 1 is satisfied. Then, for any point $q \in M$ there exists an ample $\lambda \in T^{*} M$ such that $\pi(\lambda)=q$. Moreover the set of ample covectors is open and dense in $T^{*} M$.

\section{B. Orbital diffeomorphism}

Consider two costs $L_{1}$ and $L_{2}$ in $\mathcal{L}$. We denote by $h_{1}$ and $h_{2}$ the respective normal Hamiltonians of $L_{1}$ and $L_{2}$.

Definition 3.4: We say that $\vec{h}_{1}$ and $\vec{h}_{2}$ are orbitally diffeomorphic on an open subset $V_{1}$ of $T^{*} M$ if there exists an open subset $V_{2}$ of $T^{*} M$ and a diffeomorphism $\Phi: V_{1} \rightarrow V_{2}$ such that $\Phi$ is fiber-preserving, i.e. $\pi(\Phi(\lambda))=\pi(\lambda)$, and $\Phi$ sends the integral curves of $\vec{h}_{1}$ to the integral curves of $\vec{h}_{2}$, i.e. $\Phi\left(e^{t \vec{h}_{1}} \lambda\right)=e^{t \vec{h}_{2}}(\Phi(\lambda))$ for all $\lambda \in V$ and $t \in \mathbb{R}$ for which $e^{t \vec{h}_{1}} \lambda$ is well defined, or, equivalently

$$
d \Phi \circ \vec{h}_{1}(\lambda)=\vec{h}_{2}(\Phi(\lambda)) .
$$

We say that $\Phi$ is an orbital diffeomorphism from $\vec{h}_{1}$ to $\vec{h}_{2}$. 
Proposition 3.5: If $\vec{h}_{1}$ and $\vec{h}_{2}$ are orbitally diffeomorphic on a neighborhood of $\pi^{-1}\left(q_{0}\right)$, then $L_{1}, L_{2}$ are equivalent via geodesics at $q_{0}$.

The converse statement holds near ample geodesics.

Proposition 3.6: Assume that the Lagrangians $L_{1}$ and $L_{2}$ are equivalent via geodesics at $q_{0}$. Then, for any $\lambda_{1} \in$ $\pi^{-1}\left(q_{0}\right)$ ample with respect to $L_{1}, \vec{h}_{1}$ and $\vec{h}_{2}$ are orbitally diffeomorphic on a neighborhood $V_{1}$ of $\lambda_{1}$ in $T^{*} M$.

The idea behind this result is to construct the orbital diffeomorphism near ample covectors by using the maps $P_{i}^{k_{0}}: T^{*} M \mapsto J^{k_{0}}\left(L_{i}\right)$, which are locally diffeomorphic on their image, and the identification between the geodesics (and so between their jets) of two equivalent costs:

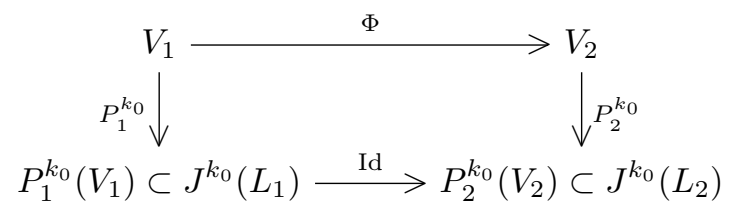

\section{Coordinates of an orbital diffeomorphism}

From Proposition 3.6, the existence of an orbital diffeomorphism is a necessary condition for the equivalence between two costs. Let us give an expression of such a diffeomorphism in well chosen local coordinates.

Consider two costs in $\mathcal{L}$,

$$
L_{1}(q, u)=u^{T} R_{1}(q) u \quad \text { and } \quad L_{2}(q, u)=u^{T} R_{2}(q) u .
$$

At each point $q \in M$ we define the transition matrix

$$
S(q)=R_{1}(q)^{-1} R_{2}(q)
$$

which is a $(m \times m)$ positive definite matrix. Thus its eigenvalues $\alpha_{1}(q), \ldots, \alpha_{m}(q)$ are positive real numbers.

Definition 3.7: A point $q_{0} \in M$ is called stable with respect to the pair $\left(R_{1}, R_{2}\right)$ if $q_{0}$ is a regular point and if the number $N(q)$ of distinct eigenvalues of $S(q)$ is constant in some neighborhood of $q_{0}$.

Notice that the set of stable points is open and dense in $M$.

Fix a point $q_{0} \in M$ which is stable with respect to $\left(R_{1}, R_{2}\right)$ and a neighborhood $U$ of $q_{0}$ containing only stable points. For $q \in U$, the matrices $R_{1}(q)$ and $R_{2}(q)$ can be simultaneously diagonalized, i.e. there exists an orthogonal matrix $P(q)$, with $q \mapsto$ $P(q)$ smooth, such that $P(q)^{T} R_{1}(q) P(q)$ is the identity matrix and $P(q)^{T} R_{2}(q) P(q)$ is a diagonal matrix with $\alpha_{1}(q), \ldots, \alpha_{m}(q)$ on the diagonal. Thus, up to a feedback transformation $u \mapsto P(q) u$, the costs $L_{1}, L_{2}$ take the form

$$
L_{1}(q, u)=\sum_{i=1}^{m} u_{i}^{2} \quad \text { and } \quad L_{2}(q, u)=\sum_{i=1}^{m} \alpha_{i}(q) u_{i}^{2} .
$$

Note that such feedback transformations are allowed since they preserve the set of geodesics.

Choose vector fields $f_{m+2}, \ldots, f_{n}$ whose values complete $f_{1}, \ldots, f_{m}, f_{m+1}=f_{0}$ into a basis of the tangent space on $U$. For all $q \in U$, the functions $T_{q}^{*} M \ni p \mapsto \nu_{i}=$ $\left\langle p, f_{i}(q)\right\rangle, i=1, \ldots, n$, define coordinates on $T_{q}^{*} M$. In these coordinates the Hamiltonians $h_{1}, h_{2}$ corresponding to the costs $L_{1}, L_{2}$ respectively can be expressed as:

$$
h_{1}=\nu_{m+1}+\frac{1}{2} \sum_{i=1}^{m} \nu_{i}^{2}, \quad h_{2}=\nu_{m+1}+\frac{1}{2} \sum_{i=1}^{m} \frac{\nu_{i}^{2}}{\alpha_{i}} .
$$

Let $\Phi$ be an orbital diffeomorphism from $\vec{h}_{1}$ to $\vec{h}_{2}$ on some neighborhood $V \subset T^{*} U$ of $\lambda_{0}$ such that $\pi\left(\lambda_{0}\right)=q_{0}$. In coordinates on $T^{*} U, \Phi$ maps $\lambda=\left(q ; \nu_{1}, \ldots, \nu_{n}\right)$ to $\left(q ; \Phi_{1}, \ldots, \Phi_{n}\right)$ where $\Phi_{i}(\lambda)=\nu_{i}(\Phi(\lambda))$.

Proposition 3.8: If $\Phi$ is an orbital diffeomorphism from $\vec{h}_{1}$ to $\vec{h}_{2}$ with coordinates $\left(\Phi_{1}, \ldots, \Phi_{n}\right)$, then:

- for $k=1, \ldots, m$, there holds $\Phi_{k}=\alpha_{k} \nu_{k}$;

- $\widetilde{\Phi}=\left(\Phi_{m+1}, \ldots, \Phi_{n}\right)$ satisfies a linear system of equations,

$$
A(\nu) \widetilde{\Phi}=b(\nu),
$$

where $A(\nu)$ is a matrix with $(n-m)$ columns and an infinite number of rows, and $b(\nu)$ is a column vector with an infinite number of rows. The components of these matrices are polynomial functions in $\nu=\left(\nu_{1}, \ldots, \nu_{n}\right)$ whose coefficients depend only on $f_{1}, \ldots, f_{n}$ for $A$, and only on $f_{1}, \ldots, f_{n}$ and $\alpha_{1}, \ldots, \alpha_{m}$ for $b$.

We do not give here the general expression of $A$ and $b$ (see [16, Chap. 4]), we will do it only in the case $m=n-1$ in Sect. V.

Remark 3.9: The fact that all the coefficients of the system (9) are polynomial in $\nu$ implies that, if there exists $\lambda_{0} \in$ $T_{q_{0}}^{*} M$ with coordinates $\nu_{0}$ for which $A\left(\nu_{0}\right)$ has a nonzero minor of size $n-m$, then the corresponding minor of $A(\nu)$ is a nonzero polynomial of $\nu$ and the matrix is injective almost everywhere on $T_{q_{0}}^{*} M$. The injectivity of $A$ implies the existence of a unique solution $\Phi$.

The matrix $A$ is strongly related with the characterization of ample covectors.

Proposition 3.10: A covector $\lambda \in T^{*} M$ is ample with respect to $L_{1}$ if and only if $A(\nu(\lambda))$ is injective.

Note that, for two proportional costs $L_{1}$ and $L_{2}=c L_{1}$ which are trivially equivalent via geodesics, all eigenvalues $\alpha_{i}$ are equal to the constant $c$. The corresponding orbital diffeomorphism is proportional to the identity, i.e. $\Phi_{i}(\nu)=$ $c \nu_{i}$ for $i=1, \ldots, n$.

Corollary 3.11: If there exists an ample $\lambda$ such that $A(\nu) \widetilde{\Phi}=b(\nu)$ admits solutions near $\nu(\lambda)$ only when $\alpha_{1}=$ $\cdots=\alpha_{m}=$ const, then the inverse optimal control problem is injective.

This result shows the way to study the injectivity of the inverse problem through the analysis of the system (9). However the latter analysis requires to introduce the notion of nilpotent approximation, for which ample covectors exist (analytic system), and the system $A(\nu) \widetilde{\Phi}=b(\nu)$ is simpler since the components of $A$ and $b$ are homogeneous polynomials.

\section{NilPOTENT APPROXIMATION}

Let us briefly present the nilpotent approximation for control-affine systems defined in [6]. See [16, Chap. 4] for the details and the proofs of the lemmas. 
Let $f_{0}, \ldots, f_{m}$ be vector fields satisfying Assumptions $1, q_{0} \in M$ be a regular point, and $U$ be a neighborhood of $q_{0}$ with only regular points. The construction relies on a graded structure defined by assigning some weights to the vector fields $f_{i}$ 's: we choose a weight 2 for $f_{0}$ and weights 1 for $f_{1}, \ldots, f_{m}$ so that the normal Hamiltonian (8) is homogeneous of weighted degree 2 . These weights induce a filtration of the Lie algebra spanned by $f_{0}, \ldots, f_{m}$ in the following way. Let us define a sequence of subspaces of the set of vector fields by

$$
\begin{aligned}
& D_{1}=\operatorname{span}\left\{f_{1}, \ldots, f_{m}\right\} \\
& D_{2}=\operatorname{span}\left\{f_{0},\left[f_{i}, f_{j}\right], f_{i}: i, j=1, \ldots, m\right\} ; \\
& D_{3}=\operatorname{span}\left\{\left[f_{0}, f_{i}\right],\left[\left[f_{0}, f_{i}\right], f_{k}\right], f_{0},\left[f_{i}, f_{j}\right], f_{i}: i, j, k\right\} ;
\end{aligned}
$$

By the weak Hörmander condition (4), there exists $r \in \mathbb{N}$ such that $D_{r}(q)=T_{q} M$ for any $q \in U$. As a consequence, the following filtration of vector spaces is well defined for $q \in U$,

$$
D_{1}(q) \subset D_{2}(q) \subset \cdots \subset D_{r}(q)=T_{q} M .
$$

The filtration defines a grading structure and weighted degrees of vector fields and functions. Moreover, by [6], there exist coordinates on a neighborhood of $q_{0}$ adapted to the grading. Based on these coordinates we can construct the vector fields $\hat{f}_{0}, \ldots, \hat{f}_{m}$, each $\hat{f}_{i}$ being the homogeneous part of the highest weighted degree in the Taylor expansion of $f_{i}$, and $\hat{f}_{i}\left(q_{0}\right)=f_{i}\left(q_{0}\right)$ for $i=0,1, \ldots, m$. Therefore, $\hat{f}_{0}$ is of weighted degree 2 and $\hat{f}_{i}$ is of weighted degree 1 for any $i=1, \ldots, m$. The vector fields $\hat{f}_{0}, \ldots, \hat{f}_{m}$ are called a nilpotent approximation of $f_{0}, \ldots, f_{m}$. We can construct a frame $f_{1}, \ldots, f_{n}$ of $T M$ adapted to the filtration (10) and the corresponding frame of the nilpotent approximation $\hat{f}_{1}, \ldots, \hat{f}_{n}$ which consist of homogeneous polynomial vector fields on $\mathbb{R}^{n}$, each $\hat{f}_{i}$ being of weighted degree $k$.

Let $A$ and $b$ be the matrices from (9) corresponding to the adapted frame $f_{1}, \ldots, f_{n}$ and to some positive functions $\alpha_{1}, \ldots, \alpha_{m}$. We denote by $\hat{A}$ and $\hat{b}$ the matrices corresponding to $\hat{f}_{1}, \ldots, \hat{f}_{n}$ and to the constant functions $\alpha_{1}\left(q_{0}\right), \ldots, \alpha_{m}\left(q_{0}\right)$.

Lemma 4.1: Any component $\hat{A}_{i j}$ (resp. $\hat{b}_{j}$ ) of the matrix $\hat{A}$ (resp. $\hat{b}$ ) is either zero or the homogeneous polynomial of highest weighted degree of the corresponding component $A_{i j}$ of $A$ (resp. $b_{j}$ of $b$ ). Any minor of $\hat{A}$ is either zero or the homogeneous polynomial of highest weighted degree of the corresponding minor of $A$. As a consequence the injectivity of $\hat{A}$ implies the injectivity of $A$. fies

Notice that the so-obtained nilpotent approximation satis-

$$
\operatorname{Lie}_{q_{0}}\left(f_{0}, \ldots, f_{m}\right)=\operatorname{Lie}_{q_{0}}\left(\hat{f}_{0}, \ldots, \hat{f}_{m}\right),
$$

however the following inclusion,

$$
\begin{aligned}
\operatorname{Lie}_{q}\left(\left\{\left(\operatorname{ad} \hat{f}_{0}\right)^{s} \hat{f}_{i}: s \geq 0, i=1, \ldots, m\right\}\right) \\
\subset \operatorname{Lie}_{q}\left(\left\{\left(\operatorname{ad} f_{0}\right)^{s} f_{i}: s \geq 0, i=1, \ldots, m\right\}\right),
\end{aligned}
$$

may be strict, and in this case the weak Hörmander condition (4) does not hold for the nilpotent approximation. We can ensure this condition by introducing a supplementary assumption on the vector fields $f_{0}, f_{1}, \ldots, f_{m}$.

Assumption 2: For $q$ in an open and dense subset of $M$, there holds

$$
f_{0}(q) \in \operatorname{span}\left\{f_{i},\left[f_{i}, f_{j}\right]: i, j=1, \ldots, m\right\}(q) .
$$

To simplify the notations, we modify the definition of a regular point by requiring also the condition (11) (the set of regular points is still an open and dense subset of $M$ ).

Lemma 4.2: If $f_{0}, \ldots, f_{m}$ satisfy Assumptions 1 and 2, then the nilpotent approximation $\hat{f}_{0}, \ldots, \hat{f}_{m}$ satisfies Assumption 1.

This allows to prove the existence of ample geodesics, a result that has its own importance beyond the applications to inverse optimal control.

Theorem 4.3: Let $f_{0}, \ldots, f_{m}$ be vector fields satisfying Assumptions 1 and 2 , and $L \in \mathcal{L}$. At any regular point $q \in$ $M$ the control-affine system (1) admits a covector $\lambda \in T_{q}^{*} M$ ample with respect to $L$.

Proof: First, up to feedback equivalence, we can assume $L=u^{T} u$. Consider the nilpotent approximation at a regular point $q$ with the cost $L$, which are both algebraic and thus analytic. From Lemma $4.2, \hat{f}_{0}, \ldots, \hat{f}_{m}$ satisfies Assumption 1, hence by Theorem 3.3 there exist $\lambda \in T_{q}^{*} M$ ample with respect to $L$ for the nilpotent approximation. By Proposition 3.10, we get that $\hat{A}(\lambda)$ is injective, which implies that $A(\lambda)$ itself is injective by Lemma 4.1. We conclude by Proposition 3.10.

\section{Application to the CASE $m=n-1$}

As a first application of the approach developed in this paper we study the case where $m=n-1$. Let $f_{0}, \ldots, f_{m}$, with $m=n-1$, be vector fields satisfying Assumptions 1 and 2 , and let $L_{1}, L_{2}$ be two costs in $\mathcal{L}$. Near a stable point $q_{0}$, we can assume up to a feedback transformation that $L_{1}, L_{2}$ are of the form (7).

Proposition 5.1: Assume the costs $L_{1}, L_{2}$ to be non proportional and equivalent via geodesics in a neighborhood of a stable point $q_{0}$. Then the control-affine problem corresponding to the nilpotent approximation $\hat{f}_{0}, \ldots, \hat{f}_{m}$ at $q_{0}$ and to $L_{1}$ admits a product structure.

Proof: By Theorem 4.3, there exists an ample covector in $T_{q_{0}}^{*} M$, and thus by Prop. 3.6 there exists an orbital diffeomorphism $\Phi$ from $\vec{h}_{1}$ to $\vec{h}_{2}$. As a consequence the linear system (9) admits a solution near the ample covectors, which implies that the rank of $\left(\begin{array}{ll}A & b\end{array}\right)$ is smaller than $n-m+1$. By Lemma 4.1, the rank of $\left(\begin{array}{ll}\hat{A} & \hat{b}\end{array}\right)$ is smaller than $n-m+1$ as well, and thus the system $\hat{A} \widetilde{\Phi}=\hat{b}$ admits a solution.

Let us study this system. Since $n=m+1$, it contains only one unknown variable $\Phi_{m+1}=\Phi_{n}$. Hence $\hat{A}$ is a column vector, as $\hat{b}$, and $\hat{A} \widetilde{\Phi}=\hat{b}$ writes as

$$
\hat{A}_{j} \Phi_{n}=\hat{b}_{j}, \quad j \in \mathbb{N} .
$$


The induction formula defining $\hat{A}$ and $\hat{b}$ is given in [16, Prop. 4.10]. Note that, with our assumptions, we can take $f_{1}, \ldots, f_{m}, f_{n}=f_{0}$ as a frame adapted to the filtration (10) and we define coefficients $\hat{c}_{i j}^{k}$ by

$$
\left[\hat{f}_{i}, \hat{f}_{j}\right]=\sum_{k=1}^{n} \hat{c}_{i j}^{k} \hat{f}_{k}, \quad i, j=1, \ldots, m .
$$

With these notations, the first components of $\hat{A}$ and $\hat{b}$ are

$$
\hat{A}_{j}=\sum_{i=1}^{m} \hat{c}_{i j}^{k} \nu_{i}, \quad \hat{b}_{j}=\hat{\alpha}_{j} \hat{A}_{j} \nu_{n}, \quad j=1, \ldots, m,
$$

where $\hat{\alpha}_{j}=\alpha_{j}\left(q_{0}\right)$. Then

$$
\Phi_{n}=\hat{\alpha}_{j} \nu_{n}
$$

for any $j$ such that $\hat{A}_{j} \neq 0$. From Assumptions 1 and 2, at least one coefficient $\hat{c}_{i j}^{m+1}$ is nonzero. Therefore there exists $j^{*}$ such that $\hat{A}_{j^{*}} \neq 0$ and $\Phi_{n}=\hat{\alpha}_{j^{*}} \nu_{n}$. This implies that, for any $j=1, \ldots, m$ such that $\hat{\alpha}_{j} \neq \hat{\alpha}_{j *}$, we have $\hat{A}_{j}=0$ and thus $\hat{c}_{i j}^{n}=0$ for any $i=1, \ldots, m$. On the other hand, the nilpotent approximation being homogeneous, $k<n$ implies $\hat{c}_{i, j}^{k}=0$. As a consequence, if $\hat{\alpha}_{i} \neq \hat{\alpha}_{j^{*}}$ then $\left[\hat{f}_{i}, \hat{f}_{j}\right]=0$ for any $j=1, \ldots, n$.

Let us define local coordinates $x=\left(x_{1}, \ldots, x_{n}\right)$ as the inverse of the local diffeomorphism

$x \mapsto \exp \left(x_{n} \hat{f}_{0}+\sum_{\left\{i: \hat{\alpha}_{i}=\hat{\alpha}_{j^{*}}\right\}} x_{i} \hat{f}_{i}\right) \circ \exp \left(\sum_{\left\{i: \hat{\alpha}_{i} \neq \hat{\alpha}_{j^{*}}\right\}} x_{i} \hat{f}_{i}\right)$, and decompose $x$ as $x=\left(\bar{x}_{1}, \bar{x}_{2}\right)$, where $\bar{x}_{1}=\left(x_{n},\left\{x_{i}\right.\right.$ : $\left.\left.\hat{\alpha}_{i}=\hat{\alpha}_{j^{*}}\right\}\right)$ and $\bar{x}_{2}=\left(\left\{x_{i}: \hat{\alpha}_{i} \neq \hat{\alpha}_{j^{*}}\right\}\right)$. Note that the $\hat{\alpha}_{i}$ 's are not all equal to each other since $L_{1}$ and $L_{2}$ are assumed to be non proportional. In these coordinates, the control-affine system defined by the nilpotent approximation takes the form

$$
\left\{\begin{array}{l}
\dot{\bar{x}}_{1}=\hat{f}_{0}\left(\bar{x}_{1}\right)+\sum_{\left\{i: \hat{\alpha}_{i}=\hat{\alpha}_{j^{*}}\right\}} u_{i} \hat{f}_{i}\left(\bar{x}_{1}\right), \\
\dot{\bar{x}}_{2}=\sum_{\left\{i: \hat{\alpha}_{i} \neq \hat{\alpha}_{j^{*}}\right\}} u_{i} \hat{f}_{i}\left(\bar{x}_{2}\right) .
\end{array}\right.
$$

This dynamics is of the form (5) and together with the cost

$$
L=\sum_{\left\{i: \hat{\alpha}_{i}=\hat{\alpha}_{j^{*}}\right\}} u_{i}^{2}+\sum_{\left\{i: \hat{\alpha}_{i} \neq \hat{\alpha}_{j^{*}}\right\}} u_{i}^{2}
$$

they form a control-affine problem with a product structure, which ends the proof.

This theorem shows that, if for a given control-affine system there exists non proportional equivalent costs, then necessarily the nilpotent approximation must admit a product structure, which is a very specific condition. For instance, generically an element $\left(f_{0}, \ldots, f_{m}\right)$ in the set $\operatorname{VF}(M)^{n}$ of $n$-tuple of vector fields satisfy Assumptions 1 and 2. The product structure of the nilpotent approximation implies that the corank one distribution spanned by $f_{1}, \ldots, f_{m}$ admits as well a nilpotent approximation with product structure. Using the characterization of such distributions in [11] we get the following result.

Corollary 5.2: Assume $n=m+1$ is odd. Then for $\left(f_{0}, f_{1}, \ldots, f_{m}\right)$ in an open and dense subset of $\operatorname{VF}(M)^{n}$, the associated inverse optimal control problem is injective.

\section{CONCLUSIONS AND FUTURE WORKS}

In this paper we present a general approach to study the injectivity of the inverse optimal control problems applicable to a wide class of problems. We apply this approach to the special class of optimal control problems with $m=$ $n-1$ controls. In this case we show that a non-injective cases have the spacial structure at the level of the nilpotent approximation. We show as well that a generic control-affine problem on odd-dimensional manifold is injective.

Still in that case $m=n-1$, we plan to extend the results on the product structure obtained for the nilpotent approximation to the affine-control problem itself. A more general idea is to relate the product structure of an optimal control problem with non-injectivity of the inverse problem via decomposition of the orbital diffeomorphism in the independent components.

\section{ACKNOWLEDGMENTS}

This work was supported by a public grant as part of the Investissement d'avenir project, reference ANR11-LABX-0056-LMH, LabEx LMH, in a joint call with Programme Gaspard Monge en Optimisation et Recherche Opérationnelle, and by the Grant ANR-15-CE40-0018 of the ANR.

\section{REFERENCES}

[1] A. Agrachev, D. Barilari, and L. Rizzi. Curvature: a variational approach. Memoirs of the AMS, 1256(1225), 2018.

[2] G. Arechavaleta, J.-P. Laumond, H. Hicheur, and A. Berthoz. An optimality principle governing human walking. IEEE Transactions on Robotics, 24(1):5-14, 2008.

[3] D. Barilari and F. Boarotto. On the set of points of smoothness for the value function of affine optimal control problems. SIAM Journal on Control and Optimization, 56(2):649-671, 2018.

[4] B. Berret, E. Chiovetto, F. Nori, and T. Pozzo. Evidence for composite cost functions in arm movement planning: An inverse optimal control approach. PLOS Computational Biology, 7(10):1-18, 102011.

[5] B. Berret, C. Darlot, F. Jean, T. Pozzo, C. Papaxanthis, and J. P. Gauthier. The inactivation principle: Mathematical solutions minimizing the absolute work and biological implications for the planning of arm movements. PLOS Computational Biology, 4(10):1-25, 102008.

[6] R. Bianchini and G. Stefani. Graded approximations and controllability along a trajectory. SIAM Journal on Control and Optimization, 28(4):903-924, 1990.

[7] B. Bonnard, J.-B. Caillau, and E. Trélat. Second order optimality conditions in the smooth case and applications in optimal control. ESAIM: Control, Optimisation and Calculus of Variations, 13(2):207236, 2007.

[8] Y. Chitour, F. Jean, and P. Mason. Optimal control models of goal-oriented human locomotion. SIAM Journal on Control and Optimization, 50(1):147-170, 2012.

[9] F. Jean and S. Maslovskaya. Inverse optimal control problem: the linear-quadratic case. In 2018 IEEE Conference on Decision and Control (CDC), pages 888-893, Dec 2018.

[10] F. Jean, S. Maslovskaya, and I. Zelenko. Inverse optimal control problem: the sub-riemannian case. IFAC-PapersOnLine, 50(1):500 505, 2017. 20th IFAC World Congress.

[11] F. Jean, S. Maslovskaya, and I. Zelenko. On projective and affine equivalence of sub-riemannian metrics. Geometriae Dedicata, To appear, 2019. arXiv:1801.04257.

[12] R. E. Kalman. When is a linear control system optimal? Journal of Basic Engineering, 86(1):51-60, Mar. 1964.

[13] H. Kong, G. Goodwin, and M. Seron. A revisit to inverse optimality of linear systems. International Journal of Control, 85(10):1506-1514, 2012. 
[14] M. Kuderer, S. Gulati, and W. Burgard. Learning driving styles for autonomous vehicles from demonstration. In IEEE International Conference on Robotics and Automation (ICRA), pages 2641-2646, 2015.

[15] J. Mainprice, R. Hayne, and D. Berenson. Goal set inverse optimal control and iterative replanning for predicting human reaching motions in shared workspaces. IEEE Transactions on Robotics, 32(4):897-908, Aug 2016.

[16] S. Maslovskaya. Inverse Optimal Control : theoretical study. 2018sacly013, Université Paris-Saclay, 2018.

[17] K. Mombaur, A. Truong, and J.-P. Laumond. From human to humanoid locomotion-an inverse optimal control approach. Autonomous Robots, 28(3):369-383, Apr 2010.

[18] P. Moylan and D. B. Anderson. Nonlinear regulator and an inverse optimal control problem. IEEE Transactions on Automatic Control, 18:460- 465, 111973.

[19] A. Y. Ng and S. Russell. Algorithms for inverse reinforcement learning. In in Proc. 17th International Conf. on Machine Learning, pages 663-670. Morgan Kaufmann, 2000.

[20] J. G. Park and K. Y. Lee. An inverse optimal control problem and its application to the choice of performance index for economic stabilization policy. IEEE Transactions on Systems, Man, and Cybernetics, SMC-5(1):64-76, Jan 1975.

[21] E. Pauwels, D. Henrion, and J.-B. Lasserre. Linear conic optimization for inverse optimal control. SIAM J. Control Optim., 54(3):1798$1825,2016$.

[22] A. S. Puydupin-Jamin, M. Johnson, and T. Bretl. A convex approach to inverse optimal control and its application to modeling human locomotion. In IEEE International Conference on Robotics and Automation, pages 531-536, May 2012.

[23] N. D. Ratliff, J. A. Bagnell, and M. A. Zinkevich. Maximum margin planning. In Proceedings of the 23rd International Conference on Machine Learning, ICML '06, pages 729-736, New York, NY, USA, 2006. ACM.

[24] D. Saunders. Thirty years of the inverse problem in the calculus of variations. Reports on Mathematical Physics, 66(1):43-53, 2010.

[25] K. Sugimoto and Y. Yamamoto. Solution to the inverse regulator problem for discrete-time systems. International Journal of Control, 48(3):1285-1300, 1988.

[26] F. Thau. On the inverse optimum control problem for a class of nonlinear autonomous systems. IEEE Transactions on Automatic Control, 12(6):674-681, December 1967.

[27] E. Todorov. Optimal control theory. In D. K., editor, Bayesian Brain: Probabilistic Approaches to Neural Coding, pages 269-298, 2006.

[28] E. Todorov and M. I. Jordan. Optimal feedback control as a theory of motor coordination. Nat Neurosci, 5:1226-1235, 2002.

[29] I. Zelenko. On geodesic equivalence of riemannian metrics and sub-riemannian metrics on distributions of corank 1 . Journal of Mathematical Sciences, 135(4):3168-3194, Jun 2006. Sovrem. Mat. Prilozh. No. 21, Geom. Zadachi Teor. Upr., 79-105, 2004. 